\title{
A Mathematical Apriorist Answers Philip Kitcher
}

\section{THOMAS M. NORTON-SMITH}

Kent State University

Philip Kitcher believes it "relatively easy to rebut" the objection that his critique of mathematical apriorism presupposes too strong a notion of a priori knowledge ([1], pp. 88-9).1 After briefly rehearsing his account of a priori knowledge in the following section, I will argue in section II that the necessary condition of Kitcher's analysis is too strong. I then offer a revision of his analysis which turns out to be sympathetic to mathematical apriorism. Finally, in section III I argue that my revision is preferable by showing that Kitcher's analysis of a priori knowledge leads to an unacceptable conclusion when coupled with his liberal view of the importance of social challenges.

I begin by briefly reviewing Kitcher's account of a priori knowledge. First of all, for any knower $X$ with typical human capabilities, and for any proposition $p, a$ life is sufficient for $X$ for $p$ if and only if $X$ could have had that life and gained the minimal empirical concepts necessary to believe that $P([1], p .22)$. For example, $X$ could not believe that the sum of the angles of a euclidean triangle equals 180 degrees without first having the concept of a triangle. Given this notion of a sufficient life, Kitcher characterizes a priori knowledge as follows:

1. X knows a prior that $\mathrm{p}$ if and only if

a. $X$ knows that $P$

b. X's belief was produced by a psychological process $k$ which is an a priori warrant.

II. $k$ is an a priori warrant for $X ' s$ belief that $p$ if and only if given any life sufficient for $X$ for $P$

a. there is a psychological process $b$ which is of the same type as $k$ available for $X$ which could engender $X$ 's belief that $p$

b. if $b$ produces in $X$ the belief that $p$, then it would warrant the belief for $X$

c. if $b$ produces in $X$ the belief that $p$, then $p$.

1 P. Kitcher, The Nature of Mathematical Knowledge (Oxford, 1984). 
That is, in order for $k$ to be counted as an a priori warrant for $p$ for $X$ it must be that in all lives possible for $X$ wherein he has the kinds of cognitive capacities distinctive of humans and has gained the necessary concepts, an appropriate belief engendering, warranting and reliable psychological process of the same type as $k$ is available to $X([1], p .26)$.

Although $X$ 's characteristic human capacities must remain invariant across the possible lives of interest to Kitcher's analysis, X's intelligence may vary across those lives:

Of course, $X$ might have been more intelligent, that is, he might have had better versions of the faculties he has. We allow for this type of change. But we are not interested in lives where $X$ has extra faculties ([1], p. 26n).

In light of the foregoing qualification, we may interpret Kitcher's analysis of a priori warrants in the following way: $k$ is a priori warrant for $X$ for $p$ if and only if in all lives possible for $X$ wherein he has the cognitive capacities distinctive of humans-be they enhanced or diminished within the bounds characteristic of humans-and has gained the necessary concepts, an appropriate belief engendering, warranting and reliable psychological process of the same type is available to $X$.

Given this brief account of Kitcher's analysis, it is easy to provide the canonical argument which he often employs to defeat claims that certain psychological processes produce a priori knowledge. To defeat the claim that $k$ is an a priori warrant for $X$ for $p$, delineate a life sufficient for $X$ for $p$ wherein $X$ 's belief was engendered by a psychological process of the same type as $k$, but in that life the power of the $k$-type process to warrant $p$ for $x$ is defused by direct, theoretical or social challenges. Of course, this is not the only way that Kitcher can defeat such a claim; for a given purported a priori warrant he could show that for some sufficient life a process of the same type is warranting yet unreliable. But the bulk of his critique of mathematical apriorism is carried by instances of the above canonical argument.

Consider Sue, an individual of average intelligence, who knows on the basis of the standard mathematical proof that there is not greatest prime number. Following the proof is engaging in a certain kind of psychological process $b$ which engenders in Sue the belief that there is no greatest prime. On Kitcher's view, b does not count as an a priori warrant on any plausible account of mathematical apriorism. For example, if $b$ is a member of a type of platonic intuition, there are sufficient lives for Sue where a process indistinguishable from $b$ could engender a false belief in the truth of the unrestricted axiom of comprehension. Once more, there 
are sufficient lives which would suggest the falsity of the proposition that there is not greatest prime; it is conceivable that in some life the proof, though correct by out lights, may be counted as dubious by a majority of Sue's peers. Or perhaps direct or theoretical challenges suggest that the proposition is false in some life sufficient for Sue. Hence, there are lives sufficient for Sue in which b-type processes would not warrant the belief, violating condition (Ilb) of Kitcher's analysis. So, b is not an a priori warrant ([1], pp. 62-4).2

There is another way that Kitcher could argue against the claim that $b$ is an a priori warrant. Because Sue's intelligence can vary across lives of interest, which is reflected by allowing Sue's cognitive capacity to be either enhanced or diminished, we may suppose that there are lives in which Sue has much diminished capacities; less euphemistically, Sue is stupid in some lives. In lives where mathematical reasoning is not Sue's long suit, a b-type process will not be available to her. she may very well know that there is no greatest prime by some other means; perhaps she answered that question incorrectly on an exam, thereby learning her lesson. The existence of such possible lives wherein a b-type process is not available to engender beliefs violates condition (lla) of Kitcher's analysis, so b is not an a priori warrant.

I suggest that this result is implausible because Kitcher's necessary condition for a priori warrants is too strong. The condition is grounded by

2 Kitcher does little to specify how process types are to be demarcated, noting only that we know intuitively when some outlandish process should not be included in a type. Actually, his resistance to providing type identity criteria is methodological, for "there is probably no privileged way of dividing processes into types" ([1], p. 25). I have chosen to interpret them narrowly so that the psychological process which Sue undergoes when following the proof that there is no greatest prime is not of the same type as the process which she would undergo when (attempting) to follow a proof of the fundamental theorem of group homorphisms. That is, two processes are of the same type if they are engendered by following the same proof.

It is somewhat disappointing that Kitcher does not attempt to specify identity conditions for process types, for that specification is really quite important, especially regarding doubts about overlong proofs. Supposing that $b$ is a psychological process which results from following a simple proof that $B$ while $c$ is a process associated with the following of a complex proof that $C$. Clearly, $c$ may not warrant the belief that $C$ because of, say, reasonable doubts expressed by colleagues. If types are interpreted so broadly that both $b$ and $c$ fall under the same type, then doubts are cast on $b$ by association; if types are specified so narrowly that $b$ and $c$ fall under different types, then social challenges to c-types are not challenges to btype processes. 
the requirement that a priori warrants warrant belief against the backdrop of any experience. That is, $b$ is an a priori warrant only if no feature of any possible life influences the warranting power of $b$. If some experience should influence $b$ 's warranting power, then beliefs produced by $b$ are dependent upon experience ([1], p. 89). Now, in those lives where a b-type process is unavailable to Sue, what experience could influence the warranting power of that process? In lives where Sue's capacities are diminished the difficulty is not that some recalcitrant experience robbed the warranting power of $b ;$ it is that engaging in such a process is beyond her capabilities. Even if we agree with Kitcher that a priori warrants should warrant independent of experience, it is not clear that we want such warrants to be independent of cognitive capacity. As it stands, Kitcher's necessary condition eliminates processes which are not available in all sufficient lives, thereby prejudicing the case against processes which would have warranted in the face of any experience, had they been available.

There is an obvious and seemingly painless response which circumvents the foregoing objection. Kitcher can restrict the lives of interest in his account to those in which Sue is intelligent enough to engage in a b-type process, thereby eliminating the lives wherein she had diminished capacities, that is, lives where a b-type process is unavailable. However, I will now show that a revision of Kitcher's analysis of a priori warrants which incorporates this modification is still unsatisfactory.

Because our previous use of the notion of a life for Sue was somewhat intuitive, we begin by making it more explicit. Define a life for Sue (at time $t)$ as the total sequence of experiences that she has had (up to $t$ ). Let $U$ be the set of Sue's possible lives, and let $V$ be the set containing Sue and her counterparts ordered with respect to cognitive capacity. ${ }^{3}$ Define the set $B$, Sue's counterpart set with respect to b-type processes, as the set containing those members of $\mathrm{V}$ who have sufficient cognitive capacity to engage in a b-type process; for brevity, call B Sue's b-counterpart set.4 Define Borderline Sue to be the member of $B$ who is just intelligent enough to engage in a b-type process. That is, given Sue's actual

${ }^{3}$ Note that the use of the notion of a life $L$ in the following, where $L \in U$, differs somewhat from the earlier more intuitive usage where $L$ is an element of the cross product of $U$ and $V$.

4 The extensions of counterpart sets, of course, vary depending upon the process type under consideration. Sue is also a member of her bcounterpart set if she can engage in a b-type process.

It should be clear that working with Sue's b-counterpart set instead of the set of all of her cognitive counterparts incorporates the suggested solution to the first objection raised, i.e., that the claimed apriority of a warrant should not be rejected if a process of the same type is unavailable to a deficient Sue counterpart. 
capacities, Borderline Sue has the greatest diminished version of those capacities possible, yet can engage in a b-type process; Borderline Sue can be thought of as the minimum of $B$. Call the maximum of $B$ Enhanced Sue. Given Sue's actual capabilities, Enhanced Sue has the most enhanced version of those capacities with the bounds placed on human knowers; Enhanced Sue is not supernatural.

Now, let $E_{a} \subseteq U$ be the set of lives which would not rob a b-type process of its warranting power for actual Sue. Similarly, Let $E_{b} \subseteq U$ and $E_{e} \subseteq U$ be the sets of lives for Borderline Sue and Enhanced Sue, respectively, which would not undermine the warranting power of a b-type process. Finally, we can define such a set of lives for any $n \in B$ such that $E_{n} \subseteq U$.

There are some intuitively plausible things that can be said about the sets of lives so defined. Because actual Sue has capabilities more enhanced than Borderline Sue, there are experiences which would undermine the warranting power of a b-type process for the latter, but not for the former: Similarly, there will probably be fewer experiences that undermine the process for Enhanced Sue than for actual Sue. Thus:

(1) $\mathrm{E}_{\mathrm{b}} \subseteq \ldots \subseteq \mathrm{E}_{\mathrm{a}} \subseteq \ldots \subseteq \mathrm{E}_{\mathrm{e}} \subseteq \mathrm{U}$.

Further, it seems reasonable to suppose that if no experience could affect the power of a process to warrant belief for actual Sue, then processes of the same type would be warranting against the backdrop of any experience for any cognitively augmented counterpart of Sue.

We can employ this machinery to characterize Kitcher's necessary condition concerning a priori warrants. In order for $b$ to be an a priori warrant for Sue's belief that there is no greatest prime it must be that:

(2) $E_{b}=\ldots=E_{a}=\ldots=E_{a}=U$,

that is, regardless of the cognitive capacity of any member of Sue's bcounterpart set, in no life will a process of the b-type be undermined. By contraposition, a b-type process would not be an a priori warrant if it happens that for some $n \in B, E_{n} \subset U$. So, for example, if some experience would undermine the warranting power of a b-type process for Borderline Sue, then $b$ is not an a priori warrant for actual Sue's belief in the prime theorem, even if no experience could undermine the power of that process for actually Sue, or for every augmented Sue counterpart. So, if the E-sets were related in this manner:

(3) $\mathrm{E}_{\mathrm{b}} \subset \ldots \subset \mathrm{E}_{\mathrm{a}}=\ldots=\mathrm{E}_{\mathrm{a}}=\mathrm{U}$,

then, on Kitcher's view, $b$ is not an a priori warrant for actual Sue. 
Assuming that warranted belief which is not a priori is dependent upon experience, or a posteriori, we can derive the following sufficient condition for a posteriori warrants from Kitcher's analysis:

For any cognizer $X$, and any proposition $p$, if a psychological process $K$ warrants $X$ 's belief that $p$, but $E_{\mathbf{n}}$ $\subset U$ for some member of $X$ 's k-counterpart set, then $k$ is an a posteriori warrant for $X$ 's belief that $p$.

I submit that this is an unsatisfactory sufficient condition for a posteriori warrants, and conclude that it is so because Kitcher's necessary condition for a priori warrants is overly strong.

There are psychological processes which, as warrants, are. uncontroversially a posteriori, perceptual processes serve as a fine example. The distinguishing feature of such warrants is that regardless of an individual's cognitive endowment, there will always be an experience that could undermine the warranting power of the process-that's what it means to say that beliefs are dependent upon experience. If we were to define the E-sets for a cognizer $X$ in order to illustrate this characteristic of dependent warrants, they would be related as follows:

$$
E_{b} \subset \ldots \subset E_{a} \subset \ldots \subset E_{a} \subset U
$$

indicating that all of the E-sets are proper subsets of $U$, the set of $X$ 's possible experiences. The sufficient condition which follows form Kitcher's analysis is inadequate because it can be satisfied not only by warrants which, like perceptual processes, are clearly a posteriori, but also by processes whose E-sets are related in the manner of (3). That is, the condition derived from Kitcher's account allows some warrants which, in fact, would warrant against the backdrop of all experience for some cognitive counterpart of $X$ to be a posteriori simply because some cognitively deficient counterpart finds the process unwarranting.

Kitcher's view suggests that some processes can be "cryptodependent warrants" in the sense that they could warrant against the backdrop of any experience for some members of X's counterpart set, yet be undermined by experience for cognitively deficient members of the set. The reason behind my designation is obvious if no experience could undermine the warranting power of the process in which actual Sue engages, but some experience would subvert the process for a non-existent deficient counterpart, then the fact that the process is a dependent warrant is, so to speak, hidden. But for all practical purposes, crypto-dependent warrants are a priori warrants if one ignores all of the deficient counterparts who find the process unwarranting in some life. Ignoring these counterparts seems to be in line with the previously expressed intuition that a priori 
warrants should warrant independent of experience, not cognitive capacity. Hence, I hold that crypto-dependent warrants are a priori.

I suggested earlier that a more natural sufficient condition for a posteriori warrants could be characterized as follows:

For any cognizer $X$, and any proposition $p$, if a psychological process $k$ warrants $X$ 's belief that $p$, and $E_{n}$ $\subset U$ for all members $n$ of $X$ 's $k$-counterpart set, then $k$ is an a posteriori warrant for $X$ 's belief that $p$.

I will use this characterization to motivate a revision of Kitcher's analysis of a priori warrants. By contraposition, a necessary condition for a process $k$ being an a priori warrant is that for some member $n$ of $X$ 's k-counterpart set it is the case that $E_{n}=U$. In other words, $X$ must have at least one cognitive counterpart for whom a process of the same type cannot be undermined by experience. Because we assumed initially that there will probably be fewer experiences that subvert the process for augmented counterparts of $X$ than for actual $X$, we focus on how different experiences would affect the warranting power of the process for Enhanced $X ;$ if $E_{e} \subset U$, then for some life available to Enhanced $X$ the warrant is undermined (and will be so for every other member of $X$ 's k-counterpart set by assumption), so the process is an a posteriori warrant for $X$. However, if $E_{e}=U$, then the warrant is either a priori or crypto-dependent, hence, on my interpretation an a priori warrant for X's belief that $p$. I would therefore suggest the following analysis of a priori knowledge given that Enhanced $X$ is the maximum of $X$ 's counterpart set with respect to $X$ 's ability to engage in a $k$ type process:

1. X knows a priori that $\mathrm{p}$ if and only if

a. X knows that $P$

b. $X$ 's belief was produced by a psychological process $k$ which is an a priori warrant.

II. $k$ is an a priori warrant for $X ' s$ belief that $p$ if and only if given any sufficient life for Enhanced $X$.

a. if a k-type process produces in Enhanced $X$ the belief that $p$, then it would warrant the belief for Enhanced $X$

b. if a k-type process produces in Enhanced $X$ the belief that $p$, then $p$.

This reformulation is sympathetic to the mathematical apriorist as the following illustrates. Suppose that given Sue's actual cognitive endowment some recalcitrant social challenge could undermine the warranting power of the b-type process which, in fact, warrants her belief that there is no greatest prime. That is, $E_{a} \subset U$; the set of experiences that would not subvert the process for actual Sue is a proper subset of the set of her 
possible lives. May we decide from this that the warrant for Sue's belief is not a priori? No, for it could be crypto-dependent; perhaps for some cognitively augmented member of Sue's b-counterpart set no experience, including the social challenge to which actual Sue is susceptible, could undermine the warranting power of the process. In order to determine whether or not the b-type process which warrants her belief is cryptodependent, hence a priori, we must consider Enhanced Sue, for if the warrant is dependent it will be the case that $E_{e} \subset U$, if the warrant is a priori, then $E_{e}=U$.

\section{III}

There is further reason to accept my proposed analysis of a priori warrants over Kitcher's: his necessary condition is so strong that it is not clear that any proposition can be known a priori, for it is always possible to conceive of some sort of social challenge which would defuse the warranting power of a psychological process for some cognitive counterpart of a typical human knower. That is, Kitcher's analysis of a priori warrants, coupled with his liberal view of the importance of social challenges, entails that there probably are no a priori warrants-a welcome result for some, but seeming not for Kitcher, himself.

Kitcher takes a rather liberal view of the importance of social challenges to claims that certain beliefs are a priori because of the almost impossible task of providing an adequate criterion of significance for experiences where are relevant to assessing the apriority of beliefs. "[For the apriorist] it would be obviously futile if [such al principle of exclusion ruled out so many experiences that vast portions of our knowledge were hailed as . . . a priori" ([1], p. 89). However, placing no exclusions on experiences runs the very real risk of rendering meaningless the notion of a priori knowledge, for probably no warrants could survive the rigors of all conceivable social challenges for all of a congnizer's counterparts.

Even one of Kitcher's preferred items of a priori knowledge can be challenged with some straightforward conjuring. He asserts that:

I think that one can make a powerful case for supposing that some self-knowledge is a priori. At most, if not all, of our waking moments, each of us knows of herself that she exists ([1], p. 29).

Now, suppose that Sue knows of her own existence on the basis of a process $r$ which is type of self-reflection. Again, $r$ is an a priori warrant only if in the face of every possible social challenge the process is still warranting. Suppose further that in some sufficient life Sue finds herself a member of native tribe which holds the bizarre belief that only the tribe's deity exists, and its existence is affirmed whenever anyone is self- 


\section{A MATHEMATICAL APRIORIST ANSWERS PHILIP KITCHER 117}

reflective. Other beliefs might be naturally engendered by the process, but religious dogma teaches that these are false. Once more, the tribe openly denounces and painfully punishes all members who question this religious belicf. In the face of these kinds of social pressures it is not clear that an r-type process could be warranting.

This story is really quite fantastic; it is so because we are convinced that no experience could ever challenge the warranting power of selfreflection. But is there any way that we could ever anticipate every possible social challenge, knowing in advance how compelling they could be for Sue?

If it is desirable to preserve the notion of a priori knowledge and the substance of a psychologistic epistemology, while avoiding the task of distinguishing relevant social challenges, then I believe that the analysis of a priori warrants that I have proposed is in order. My revision of Kitcher's view revives the notion of a priori knowledge because crypto-dependent warrants would still count as being a priori in spite of the fact that they cannot pass muster against some experiences for cognitively deficient counterparts.

Sue has agreed to illustrate. As before, Sue's r-counterpart set will contain those counterparts who have sufficient cognitive capacity to engage in an r-type process, as well as Sue herself. Now, we have seen that on Kitcher's account it is unlikely that $r$ could count as an a priori warrant for Sue's belief that she exists, for we can imagine experiences possible for Sue which would undermine the power of an r-type process to warrant. Again, these experiences come in the form of perverse social challenges.

However, on the view which I recommend, arguing that a process is not an a priori warrant because some perverse experience could undermine the r-type process for Sue, given her actual cognitive endowment, is insufficient. We need to assess whether any possible experience could affect the warranting power of self-reflection for Enhance Sue, the maximum of Sue's r-counterpart set. Unfortunately, we can only speculate about the effects of social challenges on Enhanced Sue, but we can say this much: if there's any Sue counterpart for whom the r-type process will be warranting against the backdrop of any experience, it will be Enhanced Sue. I tend to believe that Enhanced Sue could see through the natives's religious dogma. 5

From the foregoing considerations, a happy result follows for the mathematical apriorist. On my view, social challenges do not pose the kind of serious threat to apriorism which Kitcher envisions, even though on

5 One may object that Kitcher makes it too hard for a process to be an a priori warrant, but my account makes it impossible to tell when a process is an a priori warrant. This has bite, of course, only if it is necessary for us to recognize that a warrant is a priori in order for it to be a priori. 
some occasions the warranting power of a purported a priori process is undermined by experience. 